\title{
Effect of nucleotide polymorphism in cis-regulatory and coding regions on amylase activity and fitness in Drosophila melanogaster
}

\author{
H Goto ${ }^{1}$, AE Szmidt, T Yamazaki ${ }^{2}$ and $\mathrm{N}$ Inomata \\ Department of Biology, Graduate School of Sciences, Kyushu University, Fukuoka 812-8581, Japan
}

\begin{abstract}
In natural populations of Drosophila melanogaster, there are many amylase (AMY) isozymes encoded by the duplicated genes, but their adaptive significance remains unclear. One approach to elucidate this issue is to understand the molecular basis of functional differences between the allelic classes. In this study, the effects of nucleotide polymorphism in $5^{\prime}$-flanking (cis-regulatory) and coding regions on AMY activity were examined, both on glucose and starch food media and in larvae and adults, using three chimeric Amylase (Amy) genes, Amy ${ }^{c 111}$, Amy ${ }^{c 161}$ and $A m y^{f c 661}$. In this notation, the first number in the superscript indicates the sequence of the $5^{\prime}$-flnaking region (similar to $A m y^{1}$ or $A m y^{6}$ ), the second number refers to the coding region and the third number to the $3^{\prime}$-flanking region. We found that effect of
\end{abstract}

nucleotide polymorphism in the coding region differed between larvae and adults. In larvae, the coding sequence of the $A m y^{6}$ allele resulted in higher AMY activity than that of $A m y^{1}$ allele, indicating the post-transcriptional differences between them. The cis-regulatory region derived from the $A m y^{6}$ allele resulted in higher AMY activity in both larvae and adults. Thus, two fitness components, developmental time and productivity, were measured to examine whether polymorphism in the cis-regulatory region between the two alleles has an effect on them, but no significant difference was detected. We raise the implications for the evolution of subfunctionalization in multigene families.

Heredity (2005) 95, 369-376. doi:10.1038/sj.hdy.6800734; published online 24 August 2005

Keywords: Drosophila; amylase; multigene family; evolution; polymorphism; cis-regulatory region

\section{Introduction}

Since the findings of the unexpectedly high amount of protein polymorphism (allozymes) in various organisms in nature (eg Harris, 1966; Hubby and Lewontin, 1966; Lewontin and Hubby, 1966), the mechanisms explaining its maintenance have been extensively discussed. At the protein level, most observations appear to be consistent with the neutral hypothesis. As it has become easy to determine nucleotide sequences, the main interest has shifted to understanding maintenance mechanisms of DNA variation rather than those of protein polymorphism. As a result, the adaptive significance of allozyme polymorphism remains unclear.

One approach to evaluate adaptive significance of allozyme polymorphism is to understand the molecular basis of functional differences between the allelic classes. In this context, the most advanced study is Fast and Slow allozyme polymorphism of alcohol dehydrogenase (Adh) gene in Drosophila melanogaster. Association studies and experimental approaches in vivo clearly showed differences in catalytic efficiency as well as in noncoding

Correspondence: N Inomata, Department of Biology, Graduate School of Sciences, Kyushu University, 6-10-1 Hakozaki, Higashi-ku, Fukuoka 812-8581, Japan. E-mail: ninomscb@mbox.nc.kyushu-u.ac.jp

${ }^{1}$ Current address: Reserch Center for Genetic Information, Medical Institute of Bioregulation, Kyushu University, Fukuoka 812-8582, Japan ${ }^{2}$ Current address: The Research Institute of Evolutionary Biology, 2-4-28 Kamiyoga, Setagaya-ku, Tokyo 150-0098, Japan

Received 18 January 2005; accepted 19 July 2005; published online 24 August 2005 regions between the two allelic classes (eg Aquadro et al, 1986; Laurie and Stam, 1988, 1994; Choudhary and Laurie, 1991; Laurie et al, 1991; Berry and Kreitman, 1993; Stam and Laurie, 1996).

In contrast to extensive progress of the studies on allozyme polymorphism from a single locus such as the Adh gene in Drosophila, examinations of the adaptive significance of isozymes, which are proteins produced by different loci, have been lacking. Since the level of activity and protein content of an individual (strain) is the sum over all the isozymes, most experimental approaches that have been used in single-gene analysis cannot be applied directly to the products of duplicated genes. Instead, we have made use of $P$-element-mediated transformation to enable us to dissect effects of individual isozymes produced by such genes.

The starch degradation of Drosophila is one of the most extensively studied processes in the evolutionary research. The $\alpha$-amylase (AMY, EC 3.2.1.1) breaks down starch into glucose and maltose. D. melanogaster has two amylase (Amy) gene copies, composed of the Amyproximal and Amy-distal genes (Gemmill et al, 1985; Levy et al, 1985). The AMY activity is repressed by its products, glucose and maltose (Doane, 1969; Hickey and Benkel, 1982; Inomata et al, 1995a) and induced by the substrate, starch (Inomata et al, 1995a). Variation in AMY activity and food-response ability (inducibility) has been found (Matsuo and Yamazaki, 1984, Yamazaki and Matsuo, 1984, Inomata et al, 1995a). Moreover, food-response ability positively correlates with fitness components 
(Matsuo and Yamazaki, 1984; Yamazaki and Matsuo, 1984), indicating that regulatory factors controlling Amy gene expression are important for adaptation.

In natural populations of D. melanogaster, there are six major and many minor AMY isozymes (Kikkawa, 1964; Doane, 1969; Dainou et al, 1987; Inomata et al, 1995b). There are considerable differences in the level of AMY activity among strains with different isozymes (Kikkawa, 1964; Doane, 1969). Hoorn and Scharloo (1978) showed differences in the $K_{\mathrm{m}}$ (enzyme-substrate affinity, the Michaelis constant) and $V_{\max }$ (maximal activity) between $\mathrm{AMY}^{1}$ and $\mathrm{AMY}^{4,6}$ strains, and suggested some of the activity differences were due to the catalytic efficiency of the enzymes. On the other hand, Hickey (1981) showed a difference in the amount of amylase protein between high- and low-activity AMY ${ }^{1}$ variants. Yamate and Yamazaki (1999) found a positive correlation between specific activity and the amount of mRNA among strains with different AMY isozymes. On the basis of these observations, the variation of specific activity between AMY isozymes is likely to be largely explained by the differences in the amount of enzymes. The previous studies did not control the genetic backgrounds of the strains with different AMY isozymes and did not examine each isozyme and gene copy separately. Therefore, it is possible that they could not properly evaluate effects of individual isozymes. Moreover, molecular dissection of the effects of activity variation between the AMY isozymes has not been done yet.
To clarify the causes of the AMY activity differences among fly strains with different AMY isozymes, we examined the effects of differences in the nucleotide sequence between two alleles, $A m y^{1}$-distal and $A m y^{6}$ distal, of one of the duplicated Amy genes. We show that nucleotide polymorphism in both cis-regulatory and coding regions affects AMY activity. We also assessed the effect of nucleotide polymorphism in cis-regulatory region on two fitness components, developmental time and productivity.

\section{Materials and methods}

\section{Chimeric Amy genes}

Chimeric Amy genes were constructed based on $A m y^{1}$ and $A m y^{6}$ alleles, which originated from the Amy-distal gene of the KO140 and $1420 \# 1$ strains of D. melanogaster, respectively (see Inomata et al, 1995b). The chimeric Amy gene consisted of three regions: $616 \mathrm{bp}$ of $5^{\prime}$-flanking region (cis-regulatory region), $1482 \mathrm{bp}$ of coding region and $260 \mathrm{bp}$ of $3^{\prime}$-flanking region. Its structure is shown in Figure 1a. There were 16 nucleotide differences in the $5^{\prime}$ flanking region between the $A m y^{1}$ and $A m y^{6}$ alleles. In all, 21 nucleotide differences, resulting in five amino-acid changes, occurred in the coding region. First, each gene region was amplified by the PCR method using the primers and $A m y^{1}$ or $A m y^{6}$ gene as a template DNA. The PCR primers used were as follows; Notd-600,

a
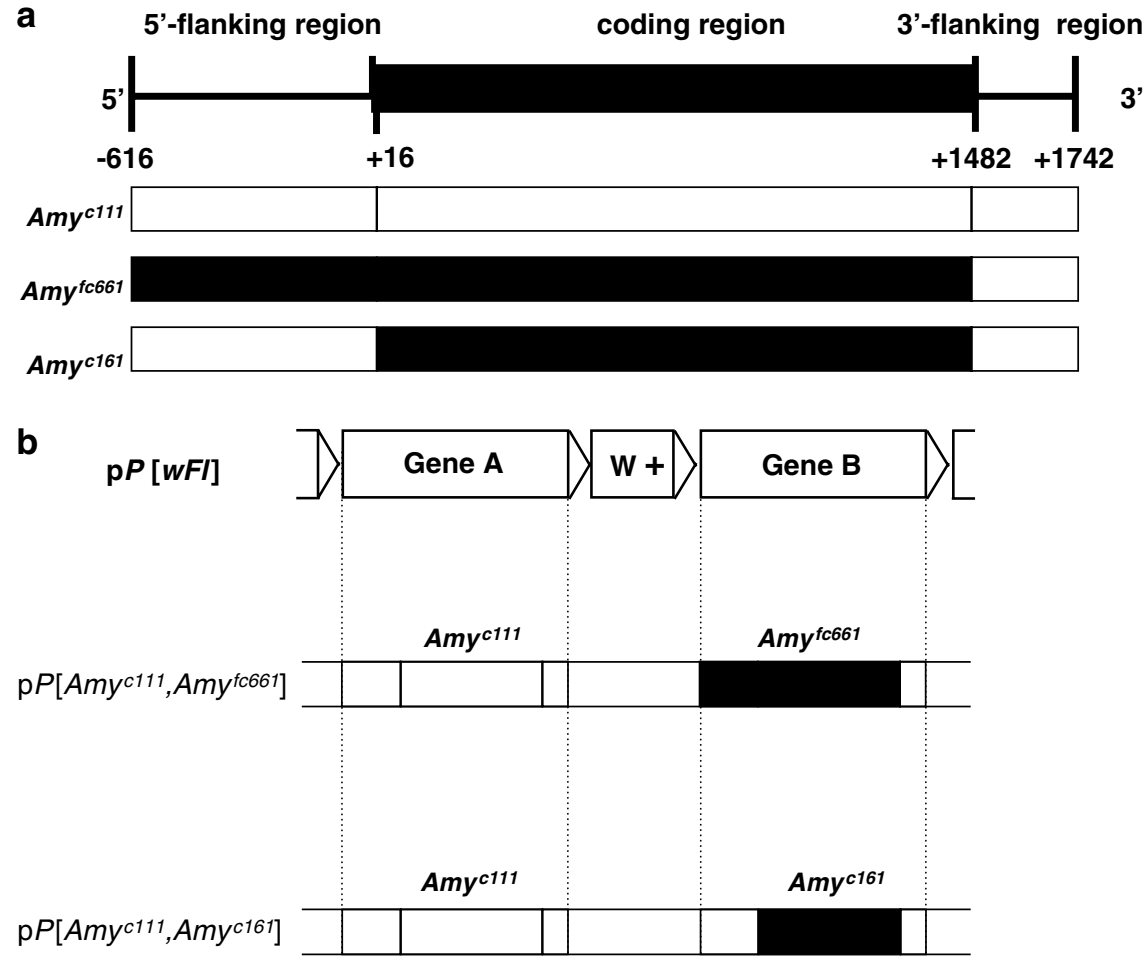

Figure 1 (a) Structure of the chimeric Amy genes. An adenine of the first codon (Met) was numbered as +1 . Numbers, $-616,+16,+1482$ and +1742 , indicate nucleotide position at which NotI, SpeI, SmaI and EcoRI restriction sites were introduced, respectively. Top: Horizontal lines indicate $5^{\prime}$ - and $3^{\prime}$-flanking regions and black bar indicates coding region. White and black boxes represent the region originated from $A m y^{1}$ and $A m y^{6}$ genes, respectively. (b) Structure of two transformation constructs, $\mathrm{p} P\left[w f l: A m y^{c 111}, A m y^{f 6661}\right]$ and $\mathrm{p} P\left[w f l: A m y^{c 111}, A m y^{c 161}\right]$, used in this study. The chimeric Amy genes were tandemly inserted into the pP[wfl] vector. Gene A indicates the Amy ${ }^{c 111}$ gene in both the $A m y^{f c 661}$ and $A m y^{c 161}$ strains. Gene B indicates the Amy $y^{f 661}$ and $A m y^{c 161}$ genes in the Amy ${ }^{c 661}$ and Amy ${ }^{c 161}$ strains, respectively. $\mathrm{w}+$ indicates mini-white gene in the vector. White and black boxes represent the region originated from $A m y^{1}$ and $A m y^{6}$ genes, respectively. 
5'-CGGGCGGCCGCTGTAGCGTGAGATTCCTAA- ${ }^{\prime}$ and Sped + , 5'-GTCACTAGTGTGCCTCGCCCT-3' for amplification of the $5^{\prime}$-flanking region, Sped-, 5'-CCGAC TAGTGACTTGGCCAGAAACATG- $3^{\prime}$ and Sma + 1500, $5^{\prime}$-TCCCCCGGGACAACTGGGGCAAAGACC-3' for amplification of the coding region, and Sma $+1485,5^{\prime}-$ TCCCCCGGGTTACAACTTGGCGTTGAC-3' and Eco + 1730, 5'-CCGGAATTCATCACGCTGAATACCGAG-3' for amplification of the $3^{\prime}$-flanking region. Each primer contains a restriction enzyme site that enables us to make any combinations of $A m y^{1}$ - or $A m y^{6}$-originated regions in the $5^{\prime}$-flanking, coding and $3^{\prime}$-flanking regions. NotI, SpeI, SmaI and EcoRI restriction sites were introduced at nucleotide positions $-616,+16,+1482$ and +1742 , respectively (the numbering scheme starts at +1 at the adenine of the first codon (Met)). Note that introduction of the SpeI site results in one amino-acid replacement (Ile to Leu) at the seventh amino-acid position. It is considered that this replacement has no effect on AMY activity, since it is located in the region coding for signal peptide (Boer and Hickey, 1986). The PCR fragments were subcloned into pGEM T-easy cloning vector (Promega), and then sequenced to check for PCR errors. Each region was combined with restriction enzyme sites, and three chimeric Amy genes, Amy $y^{c 111}, A m y^{c 161}$ and $A m y^{f c 661}$, were constructed (Figure 1a). The Amy ${ }^{c 111}$ gene contains cis-regulatory, coding and $3^{\prime}$-flanking regions originated from the Amy ${ }^{1}$ gene. Similarly, the Amy ${ }^{c 161}$ gene contains coding region originated from the $A m y^{6}$ gene and the other regions from the $A m y^{1}$ gene. The $A m y^{f c 661}$ gene contains $3^{\prime}$-flanking region originated from the $A m y^{1}$ gene and the other regions from the $A m y^{6}$ gene.

\section{Plasmid constructs}

For P-element-mediated germline transformation of $D$. melanogaster, chimeric Amy genes were introduced into the $\mathrm{p} P[w F l]$ vector (Siegal and Hartl, 1996). It is well known that expression among transformants of the same construct differs according to its genomic position, the so-called position effect (Spradling and Rubin, 1983; Laurie-Ahlberg and Stam, 1987; Brennan and Dickinson, 1988; Wilson et al, 1990). However, we overcame the problem by introducing two chimeric Amy genes into the $p \mathrm{P}[w F l]$ vector (see Figure $1 \mathrm{~b}$ ) to simultaneously insert the two constructs into a particular genomic position. In this study, the Amy c111 gene was used as a genomicposition control gene, and the $A m y^{c 161}$ or $A m y^{f 661}$ genes were used as test genes. Two transformation constructs, $\mathrm{p} P\left[w f l: A m y^{c 111}, A m y^{f c 661}\right]$ and $\mathrm{p} P\left[w f l: A m y^{c 111}, A m y^{c 161}\right]$, were generated as shown in Figure 1b.

\section{Drosophila transformation and genetic manipulations}

$P$-element-mediated transformation of D. melanogaster was carried out using the standard method (Rubin and Spradling, 1982; Spradling and Rubin, 1982) with helper plasmid $\mathrm{p} \pi \mathrm{wc}$ (Nitasaka, personal communication) and an isogenic host strain $w^{1118}$, which was full-sib mated for 14 generations. Hereafter, we denote this host strain as $w$-sib14. Three independent transformants with $P[w f l$ : $\left.A m y^{c 111}, A m y^{c 161}\right]$ insertion and two independent transformants with $P\left[w f l: A m y^{c 111}, A m y^{f c 661}\right]$ insertion were obtained. Since the Amy genes are located on the second chromosome and the host strain has $A M Y^{1,3}$ isozymes, the second chromosome of the transformants was finally replaced by the Amy ${ }^{\text {null }}$ chromosome. The transformants containing the insertion on the third chromosome were used in this study. To increase the number of independent transformants containing insertion on the third chromosome, the insertion on X and second chromosome were mobilized to new location through genetic cross using the $w ; C y O / S p ; T M 3, U b x / S b P\left[r y^{+} \Delta 2-3\right](99 B)$ strain as source of transposase $\left(P\left[r y^{+} \Delta 2-3\right](99 B)\right.$, Robertson et al, 1988). Following transformation and mobilization, all strains were crossed to the $w$-sib14; $c$ Amy $y^{\text {null }} ; \mathrm{TM} 3, \mathrm{Sb}, \mathrm{Ser} / \mathrm{Dr}$ strain to exchange genetic background. Finally, we obtained transformants containing insertion on the third chromosome with the isogenic genetic background (w-sib14 for the $\mathrm{X}$ and third chromosomes, and c Amy null for the second chromosome). This included eight and nine independent lines of the Amy $y^{c 111, f c 661}$ and $A m y^{c 11, c 161}$ strains, respectively. Hereafter, we call the former and latter the $A m y^{f c 61}$ and Amy $y^{c 161}$ strains, respectively.

\section{Food media}

Two different food media, glucose and starch, were used in this study. Glucose medium contains glucose as a carbohydrate source, and starch medium contains starch as a carbohydrate source. The components of food media were $5 \%$ ebios (killed yeast) $(\mathrm{w} / \mathrm{v}), 0.6 \%$ agar $(\mathrm{w} / \mathrm{v})$, $0.4 \%$ propionic acid (v/v) and $10 \%$ specific carbohydrate source, glucose or starch. Cornmeal medium was used to maintain stocks. Its components were $7 \%$ cornmeal $(\mathrm{w} / \mathrm{v}), 10 \%$ glucose $(\mathrm{w} / \mathrm{v}), 5 \%$ ebios $(\mathrm{w} / \mathrm{v}), 0.6 \%$ agar $(\mathrm{w} / \mathrm{v})$ and $0.4 \%$ propionic acid $(\mathrm{w} / \mathrm{v})$.

\section{Sample collection for AMY activity assay}

Five male and five female adult flies were transferred to a new vial containing each test food medium, and allowed to lay eggs at $25^{\circ} \mathrm{C}$. After 3 days, adults were collected from the vials, and then frozen at $-80^{\circ} \mathrm{C}$. After three additional days, three third-instar larvae, chosen at random, were collected from the vials without distinguishing sexes, washed with distilled water, and then kept at $-80^{\circ} \mathrm{C}$. Three replicates of larvae and adults were prepared per line for each test food medium.

\section{Measurement of AMY activity}

In this study, AMY activity was represented by the intensity of AMY isozyme bands separated on acrylamide gels. Three larvae and three adult flies were sampled without distinguishing the sexes. They were homogenized with $100 \mu \mathrm{l}$ of a buffer $(\mathrm{pH}$ 8.9) $(0.1 \mathrm{M}$ Tris-borate, $5 \mathrm{mM} \mathrm{MgCl}_{2}$ and $10 \%$ sucrose $\left.(\mathrm{w} / \mathrm{v})\right)$ by sonication. The homogenates were centrifuged at $10000 \mathrm{rpm}$ for $5 \mathrm{~min}$ and the supernatants were used for assay. To equalize samples applied to the gels, the protein content of each sample was quantified using the commercially available kit, BCA protein assay reagent (Pierce). The procedure essentially followed the manufacturer's protocol. In total, $1 \mathrm{ml}$ of the BCA working reagent made from reagent $A$ (sodium carbonate, sodium bicarbonate, BCA detection reagent and sodium tartrate in $0.1 \mathrm{~N}$ sodium hydroxide) and reagent $\mathrm{B}(4 \%$ copper sulfate solution) in the ratio of 50:1 was added to $25 \mu \mathrm{l}$ distilled water and $25 \mu \mathrm{l}$ of the supernatant. After incubation for $30 \mathrm{~min}$ at $37^{\circ} \mathrm{C}$, the absorbance was measured at $562 \mathrm{~nm}$ using spectrophotometer. 
The samples with equal protein content $(0.52 \mathrm{mg})$ were applied to the polyacrylamide gels (5\% acrylamide $(\mathrm{w} / \mathrm{v}), 0.2 \%$ bis-acrylamide (w/v), $20 \mathrm{mM} \mathrm{CaCl} 2$ and $0.1 \mathrm{M}$ Tris-borate) in a $0.1 \mathrm{M}$ Tris-borate ( $\mathrm{pH} 8.9$ ) buffer. After running for $3 \mathrm{~h}$ at $4{ }^{\circ} \mathrm{C}$ under $300 \mathrm{~V}$ constant condition, the gel was kept in starch solution (2\% soluble starch (w/v), $0.1 \mathrm{M}$ Tris- $\mathrm{HCl}(\mathrm{pH} 7.4)$ and $20 \mathrm{mM} \mathrm{CaCl}_{2}$ ) for $30 \mathrm{~min}$ at $37^{\circ} \mathrm{C}$. The gels were briefly washed with water, and incubated in solution without starch $(0.1 \mathrm{M}$ Tris- $\mathrm{HCl}\left(\mathrm{pH} \mathrm{7.4)}\right.$ and $20 \mathrm{mM} \mathrm{CaCl}$ ) for $30 \mathrm{~min}$ at $37^{\circ} \mathrm{C}$. Subsequently, they were briefly washed with water, and stained in $\mathrm{I}_{2}-\mathrm{KI}$ solution. Gel images were captured into computer by a scanner, and then the band intensity of $A M Y^{1}$ and $A M Y^{6}$ isozymes on a gel was measured, as an index of AMY isozyme activity, using NIH image software (http://rsb.info.nih.gov/nih-image/).

\section{Measurement of the fitness components}

Five virgin females and five males from the cornmeal food vials were crossed on starch food. Three virgin females and three males from the cornmeal food were crossed on glucose food. When the parental flies died, they were replaced by flies of the same condition. After 3 days, the parents were discarded. The developmental time index was obtained by calculating the average number of days of eclosion of F1 progeny since the time of crosses. Productivity was calculated as the ratio of the number of progeny to parent input flies. Counting for developmental time and productivity was carried out from the 9 th day to the 17th day for both food media.

\section{Data analysis}

Statistical analysis was carried out using the StatView software version 4.5. Three replicates of AMY activity and fitness components for each line were used in analyses.

The following two comparisons were performed by two-way analysis of variance (ANOVA). First, to evaluate effect of polymorphism in both $5^{\prime}$-flanking (cisregulatory) and coding regions, activity of the AMY ${ }^{6}$ isozyme produced by the chimeric $A m y^{f 661}$ gene was compared with that of $A M Y^{1}$ isozyme produced by the chimeric $A m y^{c 111}$ gene in the eight $A m y^{f c 661}$ strains. Second, to evaluate effect of polymorphism in coding region, activity of the $A M Y^{6}$ isozyme produced by the chimeric $A m y^{{ }^{161}}$ gene was compared with that of AMY ${ }^{1}$ isozyme produced by the chimeric $A m y^{c 111}$ gene in the nine $A m y^{c 161}$ strains. In these comparisons, the position effect of transgenes is canceled out or minimized, since the two genes under comparison have the same position in the genome. The ANOVA model was as follows, $Y_{i j k}=u+A_{i}+L_{j}+\left(A^{*} L\right)_{i j}+e_{i j k}$, where $Y$ is AMY activity, $u$ was the overall mean, $A_{i}$ is the $i$ th Amy allele effect (either $A m y^{1}$ or $\left.A m y^{6}\right), L_{j}$ is the $j$ th independent transformant line effect in the strain $(j=1-8$ or $1-9)$, $\left(A^{*} L\right)_{i j}$ is their interaction effect and $e_{i j k}$ is the error term. All effects were considered random except for the allele effect. The sum of the AMY ${ }^{1}$ and $A M Y^{6}$ activity was regarded as total AMY activity. The difference in the total AMY activity between the $A m y^{f c 661}$ and $A m y^{c 161}$ strains should reflect effect of polymorphism in the $5^{\prime}$-flanking (cis-regulatory) region between the chimeric $A m y^{f c 661}$ and $A m y^{c 161}$ genes. The total AMY activity was analyzed by the nested ANOVA. The model of the nested ANOVA was as follows, $Y_{i j k}=u+S_{i}+B_{i j}+e_{i j k}$, where $Y$ is total AMY activity, $u$ is the overall mean, $S_{i}$ is the $i$ th transformant strain effect (either $A m y^{f c 661}$ or $A m y^{c 161}$ strain), $B_{i j}$ was the random contribution for the $j$ th independent transformant line $(j=1-8$ or $1-9)$ within the $i$ th transformant strain and $e_{i j k}$ is the error term. The strain effect was considered as the fixed effect. As in the case of the total AMY activity, the difference in fitness between the $A m y^{c 661}$ and $A m y^{c 161}$ strains should reflect effect of polymorphism in the $5^{\prime}$-flanking (cis-regulatory) region between the chimeric $A m y^{f c 661}$ and $A m y^{c 161}$ genes. Fitness components data were analyzed by the nested ANOVA. The model of the nested ANOVA was as follows, $Y_{i j k}=u+S_{i}+B_{i j}+e_{i j k}$, where $Y$ is fitness component, $u$ is the overall mean, $S_{i}$ is the $i$ th transformant strain effect (either $A m y^{f c 661}$ or $A m y^{c 161}$ strain), $B_{i j}$ is the random contribution for the $j$ th independent transformant line $(j=1-8$ or $1-9)$ within the $i$ th transformant strain and $e_{i j k}$ is the error term. The strain effect was considered to be a fixed effect.

\section{Results}

Effect of polymorphism in both the $5^{\prime}$-flanking and coding regions on the AMY activity

To assess the effect of polymorphism on the AMY activity in both $5^{\prime}$-flanking and coding regions, we compared $A M Y^{1}$ activity with $A M Y^{6}$ activity in the eight $A m y^{f c 661}$
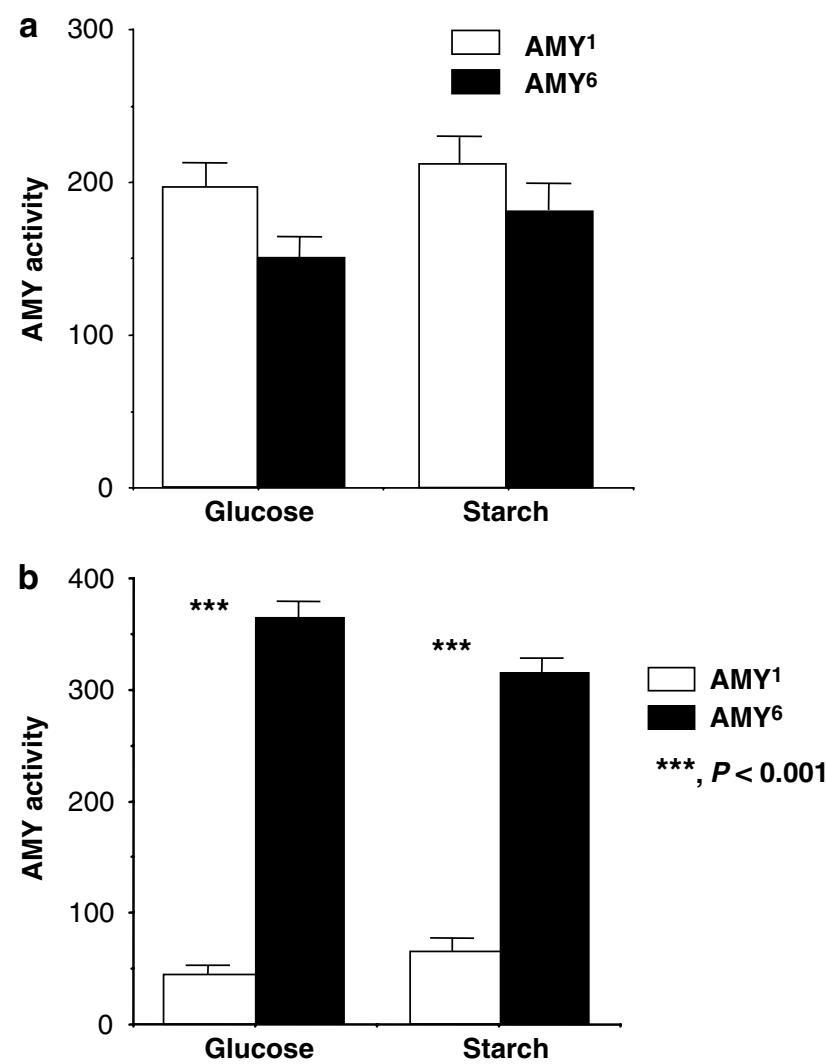

Figure 2 Effect of polymorphism on AMY activity of the $5^{\prime}$-flanking and coding regions in larvae (a) and adults (b) on glucose and starch media. White and black bars indicate average $A M Y^{1}$ and $\mathrm{AMY}^{6}$ activity, respectively, with standard error in the $A m y^{f c 661}$ strain. ${ }^{* * *}$ Indicates $P<0.001$. AMY activity was represented by the band intensity of the AMY isozyme separated on acrylamide gels. 
strains on the glucose and starch media at the larval and adult stages (Figure 2). Compared with the $\mathrm{AMY}^{1}$ activity, the $\mathrm{AMY}^{6}$ activity was slightly lower at the larval stage, but the difference was not significant on both media $\left(\mathrm{F}_{1,32}=1.844, P=0.1840\right.$ and $\mathrm{F}_{1,32}=0.284$, $P=0.5980$ on the glucose and the starch media, respectively, see Figure 2a). At the adult stage, on the other hand, the $\mathrm{AMY}^{6}$ activity was significantly higher than the AMY $^{1}$ activity on both media $\left(\mathrm{F}_{1,32}=23.655\right.$, $P<0.0001$ and $F_{1,32}=26.505, P<0.0001$ on the glucose and the starch media, respectively, see Figure $2 b$ ). Other effects were not significant at $5 \%$ level in either media at both stages. These results indicate that the effect of polymorphism on AMY activity in both the 5'-flanking and coding regions is different between the two stages.

\section{Effect of polymorphism in the coding region on the AMY activity}

To evaluate the effect of polymorphism in the coding region on the AMY activity, we compared the AMY activity with the $\mathrm{AMY}^{1}$ activity in the nine $A m y^{\text {c161 }}$ strains on the glucose and starch media at the larval and adult stages (Figure 3 ). In both media, the AMY ${ }^{6}$ activity was significantly lower than the $A M Y^{1}$ activity at the larval stage $\left(\mathrm{F}_{1,35}=77.684, P<0.0001\right.$ and $\mathrm{F}_{1,36}=54.461$,
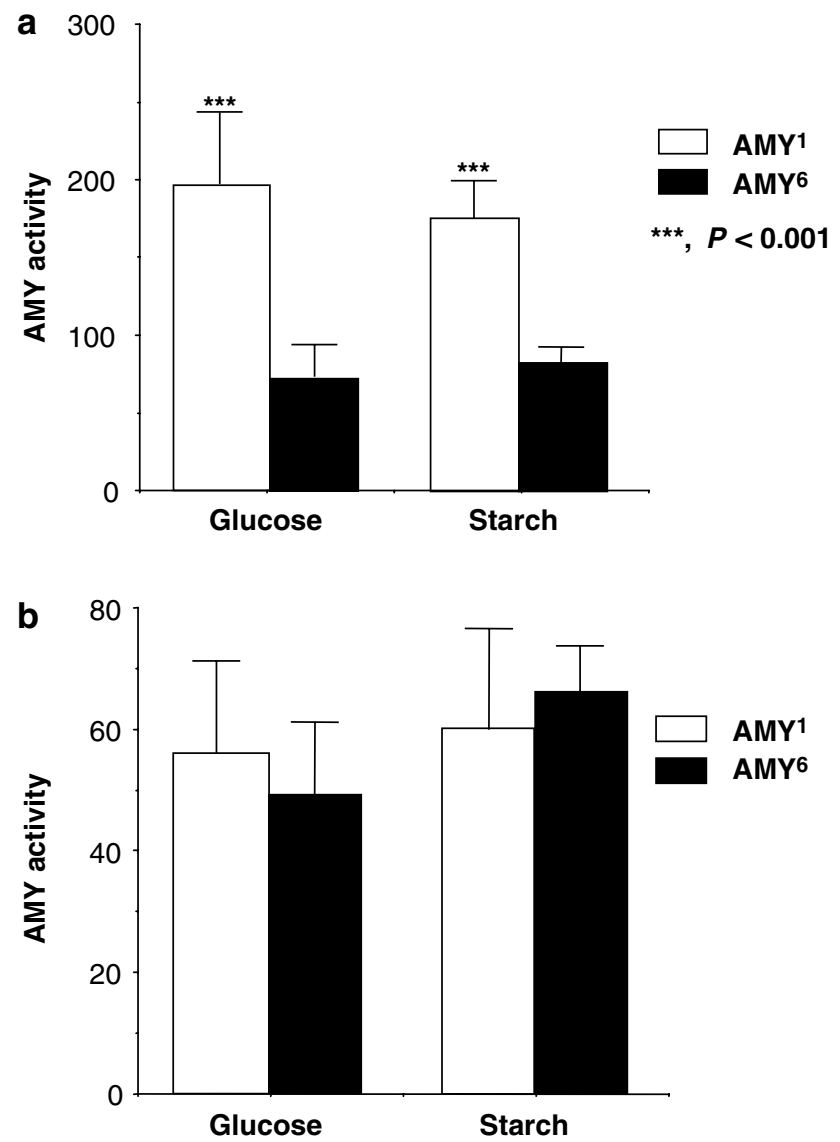

Figure 3 Effect of polymorphism on AMY activity of the coding region in larvae (a) and adults (b) on glucose and starch media. White and black bars indicate average $\mathrm{AMY}^{1}$ and $\mathrm{AMY}^{6}$ activity, respectively, with standard error in the $A m y^{c 161}$ strain. ${ }^{* * *}$ Indicates $P<0.001$. AMY activity was represented by the band intensity of the AMY isozyme separated on acrylamide gels.
$P<0.0001$ on the glucose and the starch media, respecsignificant at the adult stage on both media $\left(F_{1,36}=0.209\right.$ $P=0.6503$ and $F_{1,36}=0.075, P=0.7851$ on the glucose and the starch media, respectively, see Figure $3 b$ ). In larvae, the line effect and interaction effect between line and Amy allele were also significant in both media (line effect: $\mathrm{F}_{8,35}=17.484, P<0.0001$ and $\mathrm{F}_{8,36}=7.912$, $P<0.0001$ on the glucose and the starch media, respectively, and interaction effect: $F_{8,35}=5.455, P=0.0002$ and $\mathrm{F}_{8,36}=3.446, P=0.0048$ on the glucose and the starch media, respectively). In adults, the line effect was significant in glucose medium $\left(\mathrm{F}_{8,36}=2.231, P=0.0479\right)$. Other effects were not significant at the $5 \%$ level. These results indicate that the effect of the 21 nucleotide differences in the coding region is different between the two stages, even though the same isozymes are encoded.

Effect of polymorphism in the $5^{\prime}$-flanking region on AMY activity

To evaluate the effect of polymorphism in the $5^{\prime}$-flanking (cis-regulatory) region on the AMY activity, we compared the total AMY activity, which is the sum of $A M Y^{1}$ and $\mathrm{AMY}^{6}$ activity, between the $A m y^{f c 661}$ and $A m y^{c 161}$ strains on the glucose and starch media at the larval and adult stages (Figure 4). At the larval stage, total AMY activity
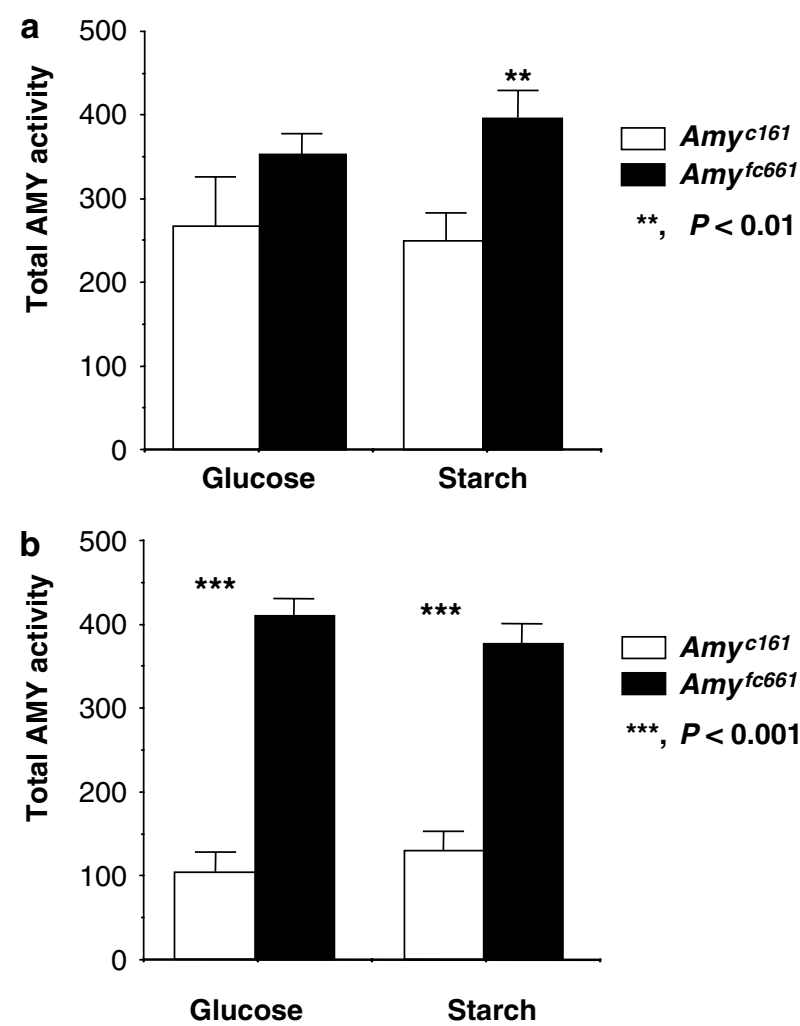

Figure 4 Effect of polymorphism on AMY activity of the $5^{\prime}$-flanking region in larvae (a) and adults (b) on glucose and starch media. White and black bars indicate average AMY activity in the Amy ${ }^{c 161}$ and $A m y^{f c 661}$ strains, respectively, with standard error. ${ }^{* *}$ and ${ }^{* * *}$ indicate $P<0.01$ and $P<0.001$, respectively. AMY activity was represented by the band intensity of the AMY isozymes separated on acrylamide gels. 
in the $A m y^{f c 661}$ strain was significantly higher than that in the $A m y^{c 161}$ strain on the starch medium $\left(\mathrm{Fs}_{1,15}=9.590\right.$, $P<0.01$, see Figure $4 \mathrm{a})$. At the adult stage, total AMY activity in the $A m y^{f c 661}$ strain was significantly higher than that in the $A m y^{c 161}$ strain on both the glucose and starch media $\left(\mathrm{Fs}_{1,15}=82.983, P<0.001\right.$ and $\mathrm{Fs}_{1,15}=67.863$, $P<0.001$ on the glucose and the starch media, respectively, see Figure $4 \mathrm{~b}$ ). Other effects were not significant at the 5\% level. These results indicate that 16 nucleotide differences in the cis-regulatory region have effect on the AMY activity between the two allelic classes.

\section{Effect of polymorphism in cis-regulatory region on fitness components}

Significant effect of polymorphism in the cis-regulatory region on AMY activity was found. Therefore, if the effect is sufficiently large, it may be possible to detect the effect on fitness. To test whether the difference in cisregulatory elements causes the difference in fitness, we measured two fitness components, developmental time and productivity, on the glucose and starch media in the $A m y^{f c 661}$ and Amy ${ }^{c 161}$ strains. Average developmental time was 13.21 and 13.13 days on glucose media in the $A m y^{f c 661}$ and $A m y^{c 161}$ strains, respectively. On the starch media, average developmental time was 12.89 and 12.72 days in the $A m y^{f c 661}$ and $A m y^{c 161}$ strains, respectively (see
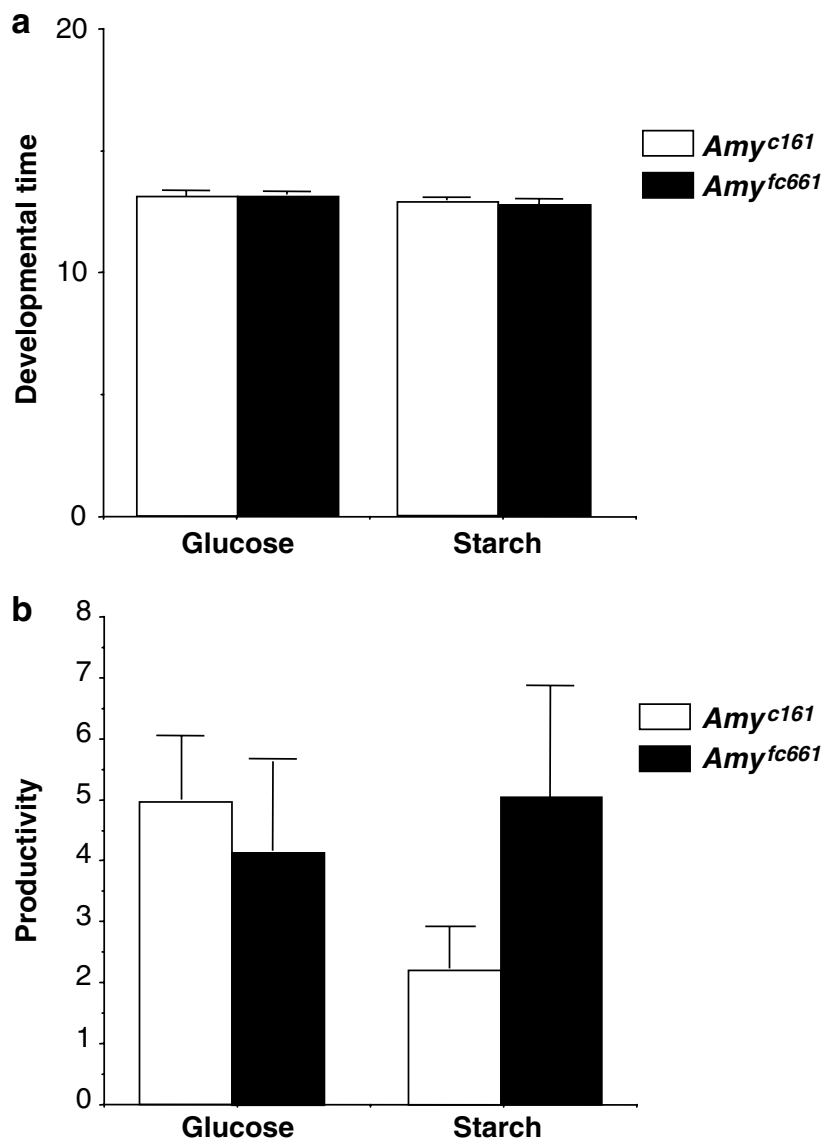

Figure 5 Effect of polymorphism on two fitness components developmental time (a) and productivity (b) on glucose and starch media. White and black bars indicate average fitness components in the $A m y^{c 161}$ and $A m y^{f c 661}$ strains, respectively, with standard error.
Figure 5a). No significant effect on developmental time was found between the $A m y^{f c 661}$ and $A m y^{c 161}$ strains on both media at $5 \%$ level $\left(\mathrm{Fs}_{1,15}=0.155\right.$ and $\mathrm{Fs}_{1,15}=0.206$ on the glucose and the starch media, respectively). Average productivity was 4.93 and 4.16 individuals on the glucose media in the Amy $y^{c 161}$ and Amy ${ }^{f c 661}$ strains, respectively. On the starch media, average productivity was 2.16 and 5.07 individuals in the $A m y^{c 161}$ and $A m y^{f c 661}$ strains, respectively (see Figure $5 b$ ). We found 2.35 times difference in productivity between the Amy fc661 and $A m y^{c 161}$ strains on the starch media at the face value. However, there was no significant effect on developmental time between the $A m y^{f 6661}$ and $A m y^{c 161}$ strains on both media at the $5 \%$ level $\left(\mathrm{Fs}_{1,15}=0.188\right.$ and $\mathrm{Fs}_{1,15}=0.240$ on the glucose and the starch media, respectively). Significant effect was found between the lines for each strain $\left(\mathrm{Fs}_{15,34}=2.2751, P<0.05\right.$ and $\mathrm{Fs}_{15,34}=9.021, P<0.001$ on the glucose and the starch media, respectively).

\section{Discussion}

\section{Effect of polymorphism on AMY activity}

The effects of the coding region variants on AMY activity were different between the larvae and adults; similarly, the combined effect of the $5^{\prime}$-flanking and coding regions differed between larvae and adults. On the other hand, the effect of polymorphism in the $5^{\prime}$-flanking region alone did not differ between the two stages. In larvae, it is likely that higher AMY activity caused by the $5^{\prime}$-flanking sequence derived from the $A m y^{6}$ allele (see Figure 4a), whereas lower AMY activity was caused by the coding sequence derived from the $A m y^{6}$ allele (see Figure 3a). The combined result was, therefore, no significant difference in AMY activity (see Figure 2a). On the other hand, in adults the higher AMY ${ }^{6}$ activity caused by the combination of $5^{\prime}$-flanking and coding sequences (see Figure 2b) is most likely to be due to the 5 '-flanking sequence (see Figure $4 \mathrm{~b}$ ), because no significant effect on AMY activity was found between the coding sequences of the two alleles (see Figure $3 b$ ). If this is true, our results suggest that effect of polymorphism in the coding region is different between larvae and adults, even though the same AMY isozymes are encoded. In other words, AMY isozymes show stage-specific activity. One plausible explanation for this observation is that the post-transcriptional processing of the mature protein, such as the stability of protein and translational efficiency, may differ between larvae and adults.

In the Amy ${ }^{c 161}$ strain, $\mathrm{AMY}^{1}$ activity was significantly higher than AMY ${ }^{6}$ activity in larvae in both glucose and starch media (see Figure 3a), indicating that the coding sequence of the $A m y^{1}$ allele results in higher AMY activity than that of the $A m y^{6}$ allele. As both the genetic background and the cis-regulatory region is the same for the chimeric genes $\left(A m y^{c 111}\right.$ and $A m y^{c 161}$ ), the amount of mRNA would be expected to be the same. Therefore, the difference in AMY activity seems to be due to the posttranscriptional differences caused by 21 nucleotide differences in the coding region of the two allelic classes. Five of these differences are nonsynonymous and may cause the difference in catalytic efficiency. Stability of mRNA, stability of protein and translational efficiency may also be affected by synonymous differences. 
The $5^{\prime}$-flanking sequence derived from the $A m y^{6}$ allele always causes higher AMY activity than that from the $A m y^{1}$ allele. This result indicates that the $A m y^{6}$-derived 5 -flanking sequence produces more mRNA, because the level of AMY activity correlates with the amount of mRNA (Hickey, 1981; Yamate and Yamazaki, 1999). There were only 16 nucleotide differences in the $5^{\prime}$ flanking region between the two alleles. No nucleotide differences were located in the core sequence of the putative cis-regulatory elements. A single-nucleotide difference may have a major effect on the level of the Amy gene expression, or alternatively, the difference in the mRNA level may be caused by nonrandom associations between polymorphism at different sites.

Since a highly inbred laboratory strain was used as a host, and assays were performed with only two allelic classes, our results might not be easily extended to other isozymes. Similarly, they might not mirror the effects of polymorphism in variable genetic backgrounds and/or under natural conditions. However, our results clearly indicate that nucleotide polymorphism in the $5^{\prime}$-flanking and coding regions appear to produce different AMY activity.

\section{Effect of polymorphism on fitness}

Yamazaki and Matsuo (1984) and Matsuo and Yamazaki (1984) showed that fitness was positively correlated with the inducibility (response ability to different food media) of AMY activity but not with activity itself. Therefore, they proposed that variation of all regulatory factors including transcriptional and post-transcriptional factors are important for adaptive evolution. The adaptive significance of changes in regulatory factors has been pointed out by several authors (eg King and Wilson, 1975). Recently, Wittkopp, Haerum and Clark (2004) indicated that cis-acting regulatory changes are more important than trans-acting ones for interspecific expression differences. In this study, we measured two fitness components, developmental time and productivity, to examine the effect of cis-acting regulatory changes. No significant effect was detected. However, on the starch media, the $A m y^{f c 661}$ strain showed 2.35 times more productivity than the $A m y^{c 161}$ strain. The difference of such magnitude may be biologically significant. Our design may have had insufficient statistical power to obtain a statistically significant result because of the small number of transformants examined.

\section{Evolutionary implications}

Some models of evolution of multigene families assume that the ancestral gene has two or more distinct but pleiotropically constrained functions (Piatigorsky and Wistow, 1991; Hughes, 1994), while other models are free of such an assumption (eg Ohno, 1970). Our results suggest that the relative AMY activity encoded by certain Amy alleles differs among the developmental stages, which indicates that they may have distinct subfunctions in Drosophila. This observation supports the model proposed by Piatigorsky and Wistow (1991) and Hughes (1994) who suggested that genes with multiple subfunctions can be important in the evolution of at least some multigene families.

\section{Acknowledgements}

We thank Dr H Araki for helpful discussion. This work was supported by the Sasagawa Scientific Research Grant from The Japan Science Society to HG, and by the Research Grant of Kato Memorial Bioscience Foundation to NI.

\section{References}

Aquadro CF, Deese SF, Bland MM, Langley CH, Laurie-Ahlberg CC (1986). Molecular population genetics of the alcohol dehydrogenase gene region of Drosophila melanogaster. Genetics 114: 1165-1190.

Berry A, Kreitman M (1993). Molecular analysis of an allozyme cline: alcohol dehydrogenase in Drosophila melanogaster on the east coast of North America. Genetics 134: 869-893.

Boer PM, Hickey DA (1986). The alpha-amylase gene in Drosophila melanogaster: nucleotide sequence, gene structure and expression motifs. Nucleic Acids Res 14: 8399-8411.

Brennan MD, Dickinson WJ (1988). Complex developmental regulation of the Drosophila affinidisjuncta alcohol dehydrogenase gene in Drosophila melanogaster. Dev Biol 125: 64-74.

Choudhary M, Laurie CC (1991). Use of in vitro mutagenesis to analyze the molecular basis of the difference in Adh expression associated with the allozyme polymorphism in Drosophila melanogaster. Genetics 129: 481-488.

Dainou O, Cariou ML, David JR, Hickey DA (1987). Amylase gene duplication: an ancestral trait in the Drosophila melanogaster species subgroup. Heredity 59: 245-251.

Doane WW (1969). Amylase variants in Drosophila melanogaster. Linkage studies and characterization of enzyme extracts. J Exp Zool 171: 321-342.

Gemmill RM, Levy JN, Doane WW (1985). Molecular cloning of alpha-amylase genes from Drosophila melanogaster. I. Clone isolation by use of mouse probe. Genetics 110: 299-312.

Harris H (1966). Enzyme polymorphism in man. Proc Roy Soc London Ser B 164: 298-310.

Hickey DA (1981). Regulation of amylase activity in Drosophila melanogaster. Variation in the number of enzyme molecules produced by different amylase genotypes. Biochem Genet 19: 783-796.

Hickey DA, Benkel B (1982). Regulation of amylase activity in Drosophila melanogaster: effects of dietary carbohydrate. Biochem Genet 20: 1117-1129.

Hoorn AJW, Scharloo W (1978). The functional significance of amylase polymorphism in Drosophila melanogaster I. Properties of two amylase variants. Genetica 49: 173-180.

Hubby JL, Lewontin RC (1966). A molecular approach to the study of genic heterozygosity in natural populations. I. The number of alleles at different loci in Drosophila pseudoobscura. Genetics 54: 577-594.

Hughes AL (1994). The evolution of functionally novel proteins after gene duplication. Proc $R$ Soc London B Biol Sci 256: $119-124$.

Inomata $\mathrm{N}$, Kanda $\mathrm{K}$, Cariou $\mathrm{ML}$, Tachida $\mathrm{H}$, Yamazaki $\mathrm{T}$ (1995a). Evolution of the response pattern to dietary carbohydrates and the developmental differentiation of gene expression of $\alpha$-amylase in Drosophila. J Mol Evol 41: 1076-1084.

Inomata N, Shibata H, Okuyama E, Yamazaki T (1995b). Evolutionary relationships and sequence variation of $\alpha-$ amylase variants encoded by duplicated genes in Amy locus of Drosophila melanogaster. Genetics 141: 237-244.

Kikkawa H (1964). An electrophoretic study on amylase in Drosophila melanogaster. Jpn J Genet 39: 401-411.

King M-C, Wilson AC (1975). Evolution at two levels in human and chimpanzees. Science 188: 107-116.

Laurie-Ahlberg GC, Stam LF (1987). Use of P-element-mediated transformation to identify the molecular basis of naturally 
occurring variants affecting Adh expression in Drosophila melanogaster. Genetics 115: 129-140.

Laurie CC, Bridgham JT, Choudhary M (1991). Associations between DNA sequence variation and variation in expression of the Adh gene in natural populations of Drosophila melanogaster. Genetics 129: 489-499.

Laurie CC, Stam LF (1988). Quantitative analysis of RNA produced by slow and fast alleles of Drosophila melanogaster. Proc Natl Acas Sci USA 85: 5161-5165.

Laurie CC, Stam LF (1994). The effect of an intronic polymorphism on alcohol dehydrogenase expression in Drosophila melanogaster. Genetics 138: 379-385.

Levy JN, Gemmill RM, Doane WW (1985). Molecular cloning of alpha-amylase genes from Drosophila melanogaster. II. Clone organization and verification. Genetics 110: 313-324.

Lewontin RC, Hubby J (1966). A molecular approach to the study of genic heterozygosity in natural populations. II. Amounts of variation and degree of heterozygosity in natural populations of Drosophila pseudoobscura. Genetics 54: 595-609.

Matsuo Y, Yamazaki T (1984). Genetic analysis of natural populations of Dorosophila melanogaster in Japan. IV. Natural selection on the inducibility, but not on the structural genes, of amylase loci. Genetics 108: 879-896.

Ohno S (1970). Evolution by Gene Duplication. Springer-Verlag: Heidelberg, Germany.

Piatigorsky J, Wistow G (1991). The recruitment of crystallins: new functions precede gene duplication. Science 252: 1078-1079.

Rubin GM, Spradling AC (1982). Transposition of cloned Pelements into Drosophila germ line chromosomes. Science 218: 341-347.
Robertson HM, Preston CR, Phillis RW, Johnson-Schlitz DM, Benz WK, Engels WR (1988). A stable genomic source of $P$ element transposase in Drosophila melanogaster. Genetics 118: 461-470.

Siegal ML, Hartl DL (1996). Transgene coplacement and high efficiency site-specific recombination with the Cre/loxP system in Drosophila. Genetics 144: 715-726.

Spradling AC, Rubin GM (1982). Genetic transposition of Drosophila germ with transposable element vectors. Science 218: 348-353.

Spradling AC, Rubin GM (1983). The effect of chromosomal position on the expression of the Drosophila xanthine dehydrogenase gene. Cell 34: 47-57.

Stam LF, Laurie CC (1996). Molecular dissection of a major gene effect on a quantitative trait: the level of alcohol dehydrogenase expression in Drosophila melanogaster. Genetics 144: 1559-1564.

Wilson C, Bellen HJ, Gehring WJ (1990). Position effects on eukaryotic gene expression. Annu Rev Cell Biol 6: 679-714.

Wittkopp PJ, Haerum BK, Clark AG (2004). Evolutionary changes in cis and trans gene regulation. Nature 430: 85-88.

Yamate N, Yamazaki T (1999). Is the difference in $\alpha$-amylase activity in the strains of Drosophila melanogaster with different allozymes due to transcriptional or posttranscriptional control. Biochem Genet 37: 345-356.

Yamazaki T, Matsuo Y (1984). Genetic analysis of natural populations of Dorosophila melanogaster in Japan. III. Genetic variability of inducing factors of amylase and fitness. Genetics 108: 223-235. 